\title{
Immunogenicity and safety of the CoronaVac inactivated vaccine in patients with autoimmune rheumatic diseases: a phase 4 trial
}

\author{
Ana C. Medeiros-Ribeiro",6, Nadia E. Aikawa ${ }^{1,2,6}$, Carla G. S. Saad', Emily F. N. Yuki ${ }^{1}$, \\ Tatiana Pedrosa ${ }^{10}$, Solange R. G. Fusco', Priscila T. Rojo', Rosa M. R. Pereira1, Samuel K. Shinjo', \\ Danieli C. O. Andrade1, Percival D. Sampaio-Barros', Carolina T. Ribeiro', Giordano B. H. Deveza', \\ Victor A. O. Martins', Clovis A. Silva², Marta H. Lopes ${ }^{3}$, Alberto J. S. Duarte ${ }^{4}$, Leila Antonangelo ${ }^{4}$, \\ Ester C. Sabino ${ }^{3,5}$, Esper G. Kallas ${ }^{3}$, Sandra G. Pasoto ${ }^{1}$ and Eloisa Bonfa ${ }^{1 凶}$
}

\begin{abstract}
CoronaVac, an inactivated SARS-CoV-2 vaccine, has been approved for emergency use in several countries. However, its immunogenicity in immunocompromised individuals has not been well established. We initiated a prospective phase 4 controlled trial (no. NCT04754698, CoronavRheum) in 910 adults with autoimmune rheumatic diseases (ARD) and 182 age- and sex-frequency-matched healthy adults (control group, CG), who received two doses of CoronaVac. The primary outcomes were reduction of $\geq 15 \%$ in both anti-SARS-CoV-2 IgG seroconversion (SC) and neutralizing antibody (NAb) positivity 6 weeks (day 69 (D69)) after the second dose in the ARD group compared with that in the CG. Secondary outcomes were IgG SC and NAb positivity at D28, IgG titers and neutralizing activity at D28 and D69 and vaccine safety. Prespecified endpoints were met, with lower anti-SARS-Cov-2 IgG SC $(70.4$ versus $95.5 \%, P<0.001)$ and NAb positivity $(56.3$ versus $79.3 \%, P<0.001)$ at $D 69$ in the ARD group than in the CG. Moreover, IgG titers (12.1 versus 29.7, $P<0.001)$ and median neutralization activity (58.7 versus $64.5 \%, P=0.013$ ) were also lower at D69 in patients with ARD. At D28, patients with ARD presented with lower IgG frequency (18.7 versus $34.6 \%, P<0.001)$ and NAb positivity $(20.6$ versus $36.3 \%, P<0.001)$ than that of the $C G$. There were no moderate/severe adverse events. These data support the use of CoronaVac in patients with ARD, suggesting reduced but acceptable short-term immunogenicity. The trial is still ongoing to evaluate the long-term effectiveness/immunogenicity.
\end{abstract}

S evere acute respiratory syndrome coronavirus 2 (SARS-CoV-2) has infected millions of people around the world ${ }^{1}$. Brazil is among those countries with the highest numbers of confirmed cases of, and deaths from, SARS-CoV-2 (refs. ${ }^{1,2}$ ), with $>430,000$ deaths registered and approximately 15 million cases as of May 2021 ( ref. $^{1}$ ). A second infection wave was driven by the Gamma coronavirus variant ${ }^{3}$, which is considered to be 2.5 -fold more contagious than the original strain ${ }^{4}$ and possibly associated with a higher risk for hospitalization and intensive care unit admission in patients younger than 60 years of age ${ }^{5}$. This second peak in March and April 2021 resulted in more than double the reported coronavirus disease 2019 (COVID-19) cases of the first peak in 2020 (ref. ${ }^{6}$ ). Vaccines are therefore essential in regard to reducing COVID-19 mortality and morbidity.

Although phase 3 clinical trials results are still being consolidated in China, Hong Kong, Indonesia, Brazil, Chile, Philippines and Turkey ${ }^{7}$, CoronaVac, an inactivated virus vaccine against SARS-CoV-2, has received emergency use approval by the World Health Organization (WHO) in several countries, including three of the six most populated in the world-Brazil, China and Turkeywhich are important for the global control of this disease. At the time of this submission, CoronaVac has accounted for approximately
$75 \%$ of the vaccines administered in Brazil. It can be kept refrigerated $^{8}$, a great advantage for deployment in developing countries. In addition, the more traditional technology using the whole virus may have the benefit of a broader immune response compared to the other vaccine platforms using only the Spike protein. This may be relevant for control of SARS-CoV-2 variants containing mutations in the Spike protein, which have been documented in Brazil ${ }^{3,9}$. Cross-reactive humoral immune responses against the Gamma and Zeta variants were achieved in healthy volunteers vaccinated with CoronaVac in a phase 3 clinical trial conducted in Brazil ${ }^{10,11}$.

However, the reported $50.7 \%$ efficacy in prevention of mild COVID-19 in the phase 3 clinical trial ${ }^{10}$ raises concerns about the immunogenicity of CoronaVac in immunosuppressed patients, who number millions, including those with autoimmune diseases, neoplasia, transplant recipients and those living with human immunodeficiency virus (HIV) among other groups, with an estimated prevalence in the United States of $2.7 \%$ of the population ${ }^{12}$. A recent letter reported a greatly reduced anti-Spike antibody response after two doses of SARS-CoV-2 mRNA 1273 or BNT162b2 vaccination in solid organ transplant recipients ${ }^{13,14}$. Previous studies on COVID-19 vaccine immunogenicity in patients with ARD have suggested slightly reduced humoral responses, but have been

'Rheumatology Division, Hospital das Clinicas HCFMUSP, Faculdade de Medicina, Universidade de Sao Paulo, Sao Paulo, Brazil. ${ }^{2}$ Pediatric Rheumatology Unit, Instituto da Criança, Hospital das Clinicas HCFMUSP, Faculdade de Medicina, Universidade de Sao Paulo, Sao Paulo, Brazil. ${ }^{3}$ Infectious Disease Department, Hospital das Clinicas HCFMUSP, Faculdade de Medicina, Universidade de Sao Paulo, Sao Paulo, Brazil. ${ }^{4}$ Central Laboratory Division, Hospital das Clinicas HCFMUSP, Faculdade de Medicina, Universidade de Sao Paulo, Sao Paulo, Brazil. ${ }^{5}$ Instituto de Medicina Tropical, Faculdade de Medicina, Universidade de

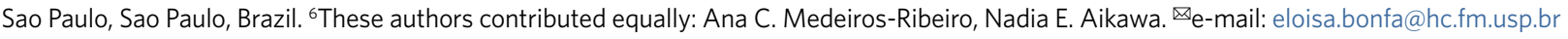


limited by the absence of a control group, small numbers of patients with $\mathrm{ARD}$, and the fact that neutralizing antibodies have not necessarily been assessed ${ }^{15-19}$. In addition, most earlier studies evaluated immunogenicity following messenger RNA vaccines and thus CoronaVac immunogenicity in immunocompromised individuals remains unclear ${ }^{13-19}$. Importantly, immunocompromised patients are at high risk for infectious diseases due to immune dysregulation and treatment regimens. In addition, they may fulfill criteria for prioritization in the context of limited vaccine supply, since COVID-19 severity is associated not only with highly prevalent comorbidities in these patients but also with disease activity ${ }^{10-24}$. Moreover, an immunocompromised state was reported to be associated with prolonged SARS-CoV-2 shedding ${ }^{25}$, reduced SARS-CoV-2 virus clearance and enhanced viral genomic evolution ${ }^{26}$, emphasizing the relevance of the vaccine for this group of patients in reducing transmission and preventing the emergence of new variants.

In this context, the present study aimed to prospectively evaluate the immunogenicity (anti-SARS-CoV-2 IgG and neutralizing antibodies) and safety of CoronaVac in a large cohort of patients with ARD compared with an age- and sex-frequency-matched control group without these conditions and with no immunosuppressive therapy. As an exploratory outcome, we further checked for incident symptomatic cases, as confirmed by real-time reverse transcriptase-PCR (RT-PCR) for SARS-CoV-2 and the presence of variants of concern (VOC) (Gamma, Alpha and Beta lineages).

\section{Results}

Study design and participants. This phase 4 prospective controlled clinical trial (CoronavRheum clinicaltrials.gov no. NCT04754698) was conducted at a single tertiary center in Brazil.

The primary outcome was humoral immunogenicity, assessed by two coprimary endpoints: a minimum of $15 \%$ reduction in SC rates of anti-S1/S2 SARS-CoV-2 IgG and the presence of NAb 6 weeks after administration of the second vaccine dose (D69) in patients with ARD compared to controls, based on a previous study of primary vaccination with the 2009 non-adjuvanted influenza A/H1N1 vaccine in a large cohort of patients with $\mathrm{ARD}^{27}$.

Secondary immunogenicity outcomes were: anti-S1/S2 IgG seroconversion and presence of NAb at D28 (after vaccine first dose); geometric mean titers of anti-S1/S2 IgG and their factor increase in geometric mean titer (FI-GMT) at D28 and D69; and median (interquartile range, IQR) neutralizing activity of NAb at D28 and D69. Another secondary outcome was safety related to the vaccine doses. Exploratory outcomes were factors associated with anti-SARS-CoV-2 IgG SC and NAb positivity at D69, and incident COVID-19 case evaluation for a total of 80 days (from day of vaccination (D0) to 10 days after the second dose (D39) and thereafter for the following 40 days (from D40 to D79)).

A total of 1,418 patients with ARD were invited to join the study, but 225 were excluded according to established criteria: acute febrile illness/symptoms of suspected COVID-19 on the day of vaccination or with real-time RT-PCR-confirmed COVID-19 $<4$ weeks before D0 $(n=24)$; demyelinating disease $(n=1)$; previous vaccination with any COVID-19 vaccine $(n=25)$; inactivated virus vaccine up to 2 weeks before D0 $(n=1)$; individuals who did not consent to participate in the study $(n=161)$; and hospitalization for general reasons $(n=13)$. Subsequently, 542 healthy adult controls were invited but 50 individuals refused to participate. The remaining 1,193 patients with $\mathrm{ARD}$ and 492 controls received the first dose of CoronaVac, but 232 (19.4\%) patients with ARD and 191 (38.8\%) controls had positive baseline IgG serology and/or $\mathrm{NAb}$ and were thus excluded from this analysis. The remaining 961 patients with ARD and 301 controls with negative serology were then frequency matched in a 5/1 ratio (five ARD/one control) by age (maximal variation \pm 5 years) and sex, with 910 patients with ARD and 182 healthy adults (CG) comprising the final study groups
Table 1 | Baseline characteristics of patients with ARD and CG

\begin{tabular}{llll} 
& $\begin{array}{l}\text { ARD } \\
(\boldsymbol{n}=910)\end{array}$ & $\begin{array}{l}\text { CG } \\
(\boldsymbol{n}=\mathbf{1 8 2})\end{array}$ & P value \\
\hline Demographics & & & \\
\hline Current age (years) & $51(40-60)$ & $50(41-60)$ & 0.985 \\
\hline Female sex & $700(76.9)$ & $140(76.9)$ & $>0.999$ \\
\hline Caucasian race & $482(53.0)$ & $82(45.1)$ & 0.051 \\
\hline Comorbidities & & & \\
\hline Systemic arterial hypertension & $400(44.0)$ & $55(30.2)$ & 0.001 \\
\hline Diabetes mellitus & $106(11.6)$ & $28(15.4)$ & 0.161 \\
\hline Dyslipidemia & $246(27.0)$ & $14(7.7)$ & $<0.001$ \\
\hline Obesity & $295(32.4)$ & $58(31.9)$ & 0.954 \\
\hline Chronic cardiomyopathy & $52(5.7)$ & $3(1.6)$ & 0.024 \\
\hline Chronic renal disease & $44(4.8)$ & 0 & 0.001 \\
\hline Current smoking & $84(9.2)$ & $21(11.0)$ & 0.461 \\
\hline Chronic obstructive pulmonary disease & $13(1.4)$ & $2(1.1)$ & $>0.999$ \\
\hline Asthma & $36(4.0)$ & $6(3.3)$ & 0.673 \\
\hline Interstitial lung disease & $78(8.6)$ & 0 & $<0.001$ \\
\hline Pulmonary hypertension & $13(1.4)$ & 0 & 0.142 \\
\hline Hematologic disease & $3(0.3)$ & 0 & $>0.999$ \\
\hline Hepatic disease & $39(4.3)$ & 0 & 0.001 \\
\hline Current cancer & $8(0.9)$ & 0 & 0.365 \\
\hline Stroke & $34(3.7)$ & 0 & 0.004 \\
\hline Current tuberculosis & $2(0.2)$ & 0 & $>0.999$ \\
\hline HIV & 0 & 0 & - \\
\hline ARD & & & \\
\hline
\end{tabular}

ARD

Chronic inflammatory arthritis

(RA, axSpA, PsA)

Other ARD (SLE, primary vasculitis, SSc, PSSj, IIM, PAPS)

Current therapy

Prednisone

451 (49.6)

$459(50.4)$

Prednisone dose, mg

$348(38.2)$

Prednisone $\geq 20 \mathrm{mg}$ day $^{-1}$

5 (5-10)

32 (3.5)

Hydroxychloroquine

Sulfasalazine

Immunosuppressive drugs

269 (29.6)

$73(8.0)$

$573(63.0)$

$229(25.2)$

Methotrexate

130 (14.3)

Leflunomide

Mycophenolate mofetil

Azathioprine

119 (13.1)

109 (12.0)

Tofacitinib

19 (2.1)

Cyclophosphamide

Tacrolimus

10 (1.1)

10 (1.1)

Cyclosporine

Biologic therapy

TNFi

Abatacept

9 (1.0)

321 (35.3)

138 (15.2)

$51(5.6)$

Tocilizumab

50 (5.5)

Belimumab

30 (3.3)

Secukinumab

29 (3.2)

Rituximab

19 (2.1)

Ustekinumab

$5(0.5)$

Results are expressed as median (IQR) and $n$ (\%). Continuous data were compared using the Mann-Whitney $U$-test, and categorical variables with the chi-square or

Fisher's exact test, as appropriate, always as two-sided analyses. 
Table 2 | Seroconversion rates at D28 and D69; anti-SARS-CoV-2 S1/S2 IgG titers before (D0) and after the first (D28) and second dose (D69) of CoronaVac vaccination in patients with ARD and CG

\begin{tabular}{|c|c|c|c|c|c|c|c|}
\hline & \multicolumn{2}{|c|}{ SC } & \multicolumn{3}{|c|}{ GMT $\left(A \cup \mathrm{ml}^{-1}\right)$} & \multicolumn{2}{|c|}{ FI-GMT } \\
\hline & D28 & D69 & DO & D28 & D69 & D0 to D28 & D0 to D69 \\
\hline$A R D, n=859$ & $161(18.7)$ & $605(70.4)$ & $2.2(2.2-2.3)$ & $5.1(4.7-5.5)$ & $27.0(24.7-29.5)$ & $2.3(2.1-2.5)$ & $12.1(11.0-13.2)$ \\
\hline$P(A R D$ versus $C G)$ & $<0.0001$ & $<0.0001$ & $>0.9990$ & $<0.0010$ & $<0.0010$ & $<0.0010$ & $<0.0010$ \\
\hline
\end{tabular}

$\mathrm{SC}$ is defined as post-vaccination titer $\geq 15 \mathrm{AU} \mathrm{ml}^{-1}$ by indirect ELISA, LIAISON SARS-CoV-2 S1/S2 lgG. Frequencies of SC are presented as number (\%), and were compared using a two-sided chi-square test between ARD and CG at prespecified time points (D28 and D69). IgG antibody titers and FI-GMT are expressed as geometric means with $95 \% \mathrm{Cl}$. Data regarding IgG titers were analyzed using ANOVA with repeated measures and two factors (two groups (ARD versus CG) at three time points (D0, D28 and D69)), followed by Bonferroni's multiple comparisons at In-transformed data (Supplementary Table 1). The behavior of IgG titers was different for ARD and CG groups between D28 and D69: mean titers increased at each time point for ARD and CG ( $P<0.001)$. FI-GMT values were compared using the Mann-Whitney U-test for intergroup comparisons in In-transformed data at prespecified time points (D28 and D69). All analyses were two-sided.

Table 3 | Frequency of NAb and median percentage of neutralizing activity in positive cases, after the first (D28) and second dose (D69) of CoronaVac vaccination in patients with ARD in comparison to CG

\begin{tabular}{|c|c|c|c|c|}
\hline & \multicolumn{2}{|c|}{ D28 } & \multicolumn{2}{|c|}{ D69 } \\
\hline & $\begin{array}{l}\text { Subjects with positive } \\
\text { NAb, } n(\%)\end{array}$ & $\begin{array}{l}\text { Neutralizing activity (\%) } \\
\text { median (IQR) }\end{array}$ & $\begin{array}{l}\text { Subjects with positive } \\
\text { NAb, } n(\%)\end{array}$ & $\begin{array}{l}\text { Neutralizing activity (\%) } \\
\text { median (IQR) }\end{array}$ \\
\hline ARD, $n=859$ & $177(20.6)$ & $42.6(35.8-60.4)$ & $484(56.3)$ & $58.7(43.1-77.2)$ \\
\hline$C G, n=179$ & $65(36.3)$ & $45(34.5-71.1)$ & $142(79.3)$ & $64.5(48.4-81.4)$ \\
\hline$P(A R D$ versus $C G)$ & $<0.0001$ & 0.4900 & $<0.0001$ & 0.0130 \\
\hline
\end{tabular}

Frequencies of subjects with positive NAb are expressed as number (\%). Positivity for NAb was defined as neutralizing activity $\geq 30 \%$ (cPass sVNT Kit). Data were compared using a two-sided chi-square test between ARD and CG at prespecified time points (D28 and D69). Percentage of neutralizing activity among subjects with positive NAb is expressed as median (IQR). Data were compared using a two-sided Mann-Whitney U-test for comparison between ARD and CG, at prespecified time points (D28 and D69).

(Extended Data Fig. 1). Enrollment and vaccination occurred on the same day for each participant. The first subject was enrolled and vaccinated on 9 February 2021 and the last participant was enrolled and vaccinated on 24February 2021. The majority $(n=1,017$, 93.1\%) of patients and controls were recruited and vaccinated on 9 or 10 February 2021, with no differences between the ARD and CG groups (92.7 versus $95.1 \%, P=0.261$ ). Patients and controls were followed until D79 after the first vaccine dose (D0) for analysis of immunogenicity and incident cases in this study. The trial is no longer recruiting, but it is still ongoing for long-term effectiveness and immunogenicity.

Patients with ARD had the following disease diagnoses: chronic inflammatory arthritis (CIA) $(n=451,49.6 \%)$, rheumatoid arthritis (RA) $(n=256,28.1 \%)$, axial spondyloarthritis (axSpA) $(n=106$, $11.6 \%)$ or psoriatic arthritis (PsA) $(n=89,9.8 \%)$ and other systemic $\operatorname{ARD}(n=459,50.4 \%)$, systemic lupus erythematosus (SLE) $(n=232$, $25.5 \%)$, primary vasculitis $(n=66,7.3 \%)$, primary Sjögren's syndrome (pSSj) $(n=42,4.6 \%)$, systemic sclerosis (SSc) $(n=41,4.5 \%)$, idiopathic inflammatory myopathies (IIM) $(n=41,4.5 \%)$ and primary antiphospholipid syndrome (PAPS) $(n=37,4.1 \%)$ (Table 1$)$. The control group $(n=182, C G)$ included hospital cleaning and general maintenance services workers $(n=109,59.9 \%)$, health professionals $(n=45,24.7 \%)$ and hospital administrative services employees or their relatives $(n=28,15.4 \%)$.

The ARD and CG groups had comparable median ages (51 versus 50 years, $P=0.985$ ) and enrollment of females (76.9 versus $76.9 \%, P>0.999)$ (Table 1). Frequencies of comorbidity were higher in ARD, particularly systemic arterial hypertension (44.0 versus $30.2 \%, P=0.001)$, dyslipidemia ( 27.0 versus $7.7 \%, P<0.001)$, interstitial lung disease $(8.6$ versus $0 \%, P<0.001)$, cardiomyopathy (5.7 versus $1.6 \%, P=0.024)$ and chronic renal disease (4.8 versus $0 \%$, $P=0.001)$ (Table 1$)$. A total of $348(38.2 \%)$ patients with ARD were receiving ongoing treatment with prednisone and 573 (63.0\%) were using immunosuppressive drugs. Of those patients treated with immunosuppressive drugs, $25.2 \%$ were using methotrexate, $14.3 \%$ leflunomide, $13.1 \%$ mycophenolate mofetil, $12 \%$ azathioprine and $<3 \%$ others. Of those $321(35.3 \%)$ patients were being treated using biologic therapies, $15.2 \%$ were using tumor necrosis factor inhibitor (TNFi), 5.6\% abatacept, 5.5\% tocilizumab, 3.3\% belimumab, 3.2\% secukinumab and $<3 \%$ others (Table 1 ).

For the primary outcome analysis of immunogenicity, we excluded $38(4.2 \%)$ participants (35 patients with ARD and three CG participants) with real-time RT-PCR-confirmed COVID-19 after either the first or second dose of vaccine until D69, and 16/910 (1.5\%) patients who did not attend the final visit (D69), including two deaths not related to COVID-19.

Primary immunogenicity outcomes. Humoral response parameters in the remaining 859 patients with ARD and 179 controls, all with negative anti-SARS-CoV-2 S1/S2 IgG antibodies and NAb prevaccination, are shown in Tables 2 and 3.

The study met the primary outcomes, defined as a minimum of $15 \%$ reduction in anti-S1/S2 SARS-CoV-2 IgG SC and in the presence of NAb in patients with ARD compared to CG at 6 weeks (D69) after the second dose. Analysis of the SARS-CoV-2 S1/S2 IgG response at D69 revealed a lower SC rate in patients with ARD (70.4 versus 95.5\%, $P<0.001)$. Similarly, NAb positivity was lower in patients with ARD compared to controls (56.3 versus $79.3 \%, P<0.001$ ).

Secondary outcomes. Secondary immunogenicity outcomes defined by anti-SARS-CoV-2 IgG SC at D28, as well as IgG GMT and FI-GMT at D28 and D69, are presented in Table 2 and Fig. 1. SARS-CoV-2 cPass virus NAb positivity at D28 and median activity of NAb at D28 and D69 were also secondary outcomes (Table 3).

A minority of participants in both groups developed anti-SARSCoV-2 IgG antibodies after the first dose (D28), with a lower frequency and level in patients with ARD compared to CG (161 $(18.7 \%)$ versus $62(34.6 \%), P<0.001)$ and FI-GMT $(2.3$ (95\% confidence interval (CI) 2.1-2.5) versus 4.6 (95\% CI 3.9-5.4), $P<0.001$ ). The SC rates doubled after the second vaccine dose, with an 


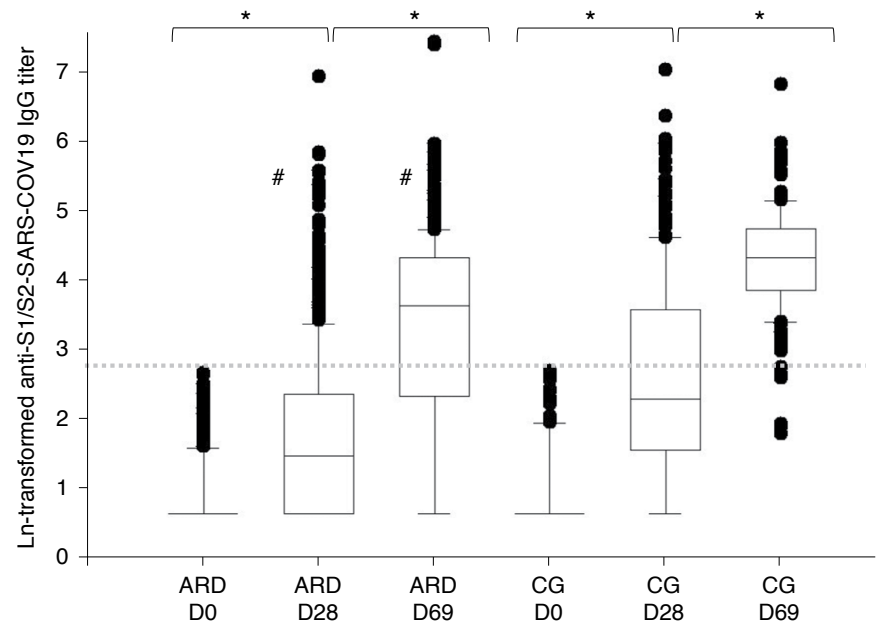

Fig. 1 | Anti-SARS-CoV-2 S1/S2 IgG titers of patients with ARD and subjects in CG at D0, D28 and D69. Box plots show the distribution of In-transformed IgG titers over time. Data for each group (ARD, $n=859$ and $C G, n=179)$ are presented at each time point as box plots: central values within boxes correspond to median (50th percentile, or Q2); the range between the lower (25th percentile, or Q1) and upper (75th percentile, or Q3) bounds of the boxes is the IQR. Whiskers represent scores outside $\mathrm{IQR}$ and ends in maximum (higher "calculated value" $=\mathrm{Q} 3+1.5 \times \mathrm{IQR}$ ) and minimum (lower "calculated value" $=\mathrm{Q} 1-1.5 \times 1 \mathrm{Q}$ ). Spots are outliers above the maximum or under the minimum values. The minimum possible value is $0.64\left(\ln 1.9\right.$, the value attributed to $\lg$ titers $\left.\leq 3.8 \mathrm{AU} \mathrm{m}^{-1}\right)$. Data regarding IgG titers were analyzed using ANOVA with repeated measures and two factors (two groups (ARD versus CG), at three time points (D0, D28 and D69)), followed by Bonferroni's multiple comparison of In-transformed data (Supplementary Table 1). Tests were always two-sided. The mean behavior of the In-transformed IgG titers was different in ARD and CG groups at D28 $(P<0.001)$ and D69 $(P<0.001)$. Mean titers increased at each time point for ARD and CG ( $P<0.001)$. At D28 and D69 evaluations, patients with ARD presented lower mean titers than CG $\left({ }^{\#} P<0.001\right)$. ARD and CG were comparable only at DO ( $\left.P>0.999\right)$. Dotted line denotes the cut-off level for positivity $\left(\ln 15 \mathrm{AU} \mathrm{ml}^{-1}=2.71\right.$ by Indirect ELISA, LIAISON SARS-CoV-2 S1/S2 lgG).

increase of more than fivefold in GMT (FI-GMT) for both groups (Table 2 and Fig. 1).

According to Bonferroni's multiple comparison, the mean behavior of the neperian logarithm (ln)-transformed IgG titers was different in the ARD and CG groups between D28 and D69 $(P<0.001)$. Mean IgG titers were similar at D0 in both groups $(P>0.999)$ and increased at each time point for ARD and CG $(P<0.001)$. At the D28 and D69 evaluations, patients with ARD presented lower mean titers than CG $(P<0.001)$ (Table 2, Fig. 1 and Supplementary Table 1).

Analysis of the dynamics of NAb detection showed that after the first dose (D28), a minority of participants had positive antibodies and patients with ARD had lower frequencies (177 (20.6\%) versus $65(36.3 \%), P<0.001)$, but with similar median (IQR) activity (42.6\% (35.8-60.4) versus 45\% (34.5-71.1), $P=0.490)$ compared with CG (Table 3). At D69, lower median (IQR) neutralization activity $(58.7 \%(43.1-77.2)$ versus $64.5 \%$ (48.4-81.4), $P=0.013)$ was observed.

Vaccine tolerance and safety. Vaccine safety analysis, another secondary outcome, is illustrated in Table 4 . No moderate/severe adverse events (AEs) related to the vaccine were reported. After the first dose, the most frequently reported vaccine reactions in patients with ARD and CG were pain at the injection site (19.8 versus $17.0 \%, P=0.388)$, headache $(20.2$ versus $11.0 \%, P=0.003)$ and somnolence (13.6 versus $10.4 \%, P=0.243$ ). Overall reactions were more frequently reported in patients with ARD than CG (50.5 versus $40.1 \%, P=0.011)$, including arthralgia (13.5 versus $6.0 \%, P=0.005$ ), back pain (9.8 versus $4.9 \%, P=0.037$ ), malaise (9.5 versus $4.4 \%, P=0.026$ ), nausea (6.1 versus $2.2 \%$, $P=0.032$ ) and sweating (5.6 versus $1.1 \%, P=0.007)$. After the second dose, patients with ARD reported less local itching $(2.7$ versus $5.5 \%, P=0.047)$ and more sweating (5.3 versus $1.1 \%$, $P=0.010)($ Table 4$)$.

Factors associated with lower anti-SARS-CoV-2 IgG SC and NAb positivity in patients with ARD. We also analyzed factors associated with anti-SARS-CoV-2 IgG SC and NAb positivity as exploratory outcomes (Table 5). Patients with negative anti-SARS-CoV-2 IgG after two doses of CoronaVac (D69) were of older age $(P<0.001)$, with a higher frequency of females $(81.9$ versus $74.7 \%$, $P=0.023)$ compared to those with positive anti-SARS-CoV-2 IgG. Non-seroconverters used the following therapies more often: prednisone (55.9 versus $31.1 \%, P<0.001$ ) and prednisone $\geq 20 \mathrm{mg} \mathrm{day}^{-1}$ (5.5 versus $2.6 \%, P=0.037$ ); immunosuppressants (81.9 versus $54.5 \%, P<0.001$ ), particularly methotrexate (34.6 versus $21.7 \%$, $P<0.001)$ and mycophenolate mofetil (24.4 versus $7.9 \%, P<0.001)$; and biologic therapy (44.1 versus $32.2 \%, P=0.001$ ), especially abatacept (11.4 versus $3.3 \%, P<0.001)$ and rituximab (4.3 versus $1.3 \%, P=0.006)$ (Table 5). Multivariate logistic regression analysis (Supplementary Table 2) was performed using as dependent variables SC or the presence of NAb at D69 (primary endpoint), and as independent variables those with $P<0.2$ in the univariate analysis presented in Table 5 . This analysis revealed that age $\geq 60$ years (odds ratio $(\mathrm{OR})=0.51 ; 95 \% \mathrm{CI} 0.36-0.74, P<0.001$ ), prednisone $(\mathrm{OR}=0.40$; $95 \% \mathrm{CI} 0.28-0.56, P<0.001)$, methotrexate $(\mathrm{OR}=0.42$; 95\% CI $0.29-0.61, P<0.001)$, mycophenolate mofetil $(\mathrm{OR}=0.15 ; 95 \% \mathrm{CI} 0.09-0.24, P<0.001), \mathrm{TNFi}(\mathrm{OR}=0.41 ; 95 \% \mathrm{CI}$ $0.26-0.64, P<0.001)$, abatacept $(\mathrm{OR}=0.24 ; 95 \% \mathrm{CI} \quad 0.13-0.46$, $P<0.001)$ and rituximab $(\mathrm{OR}=0.34 ; 95 \% \mathrm{CI} 0.13-0.93, P=0.036)$ were associated with the absence of SC in patients with ARD (Supplementary Table 2).

Similarly, patients with negative NAb after complete vaccination (D69) were older (52 (43-62) versus 49 (39-59) years, $P<0.001)$ than those with positive NAb. Patients with negative NAb at D69 were more frequently $\geq 60$ years of age ( 32.5 versus $22.5 \%, P=0.001$ ) and using prednisone ( 49.3 versus $30 \%, P<0.001$ ), immunosuppressants $(72.5$ versus $55 \%, P<0.001)$, including methotrexate (30.4 versus $21.7 \%, P=0.004)$ and mycophenolate mofetil $(17.9$ versus $8.9 \%, P<0.001$ ) or biologic therapy (41.3 versus $31.4 \%$, $P=0.003)$, including abatacept $(8.0$ versus $3.9 \%, P=0.011)$ and rituximab (4.0 versus $0.8 \%, P=0.002$ ) (Table 5 ). Multivariate analysis identified age $\geq 60$ years $(\mathrm{OR}=0.65 ; 95 \% \mathrm{CI} 0.46-0.91, P=0.011)$, prednisone $(\mathrm{OR}=0.48$; 95\% CI $0.35-0.65, P<0.001)$, methotrexate $(\mathrm{OR}=0.67,95 \% \mathrm{CI} 0.47-0.95, P=0.024)$, mycophenolate mofetil $(\mathrm{OR}=0.33$; 95\% CI $0.21-0.53, P<0.001)$ and rituximab $(\mathrm{OR}=0.28$; $95 \%$ CI $0.09-0.87, P=0.028)$ as associated with the absence of neutralizing activity in patients with ARD (Supplementary Table 2).

COVID-19 incident cases. For the analysis of incident cases, another exploratory outcome was used-participants were followed during strictly equivalent time periods of 40 days before and after full vaccination: from D0 to D39 and from D40 to D79. Therefore, the evaluation period for incident cases was extended to 10 days (D79) after the final immunogenicity analysis (D69). A total of 39 incident symptomatic, RT-PCR-confirmed COVID-19 cases among patients with ARD and CG were observed during the evaluation periods, with no significant difference between groups (4.0 versus $1.6 \%, P=0.186$ ). The frequency of cases occurring 
Table 4 | Adverse events following CoronaVac vaccination in patients with ARD and CG

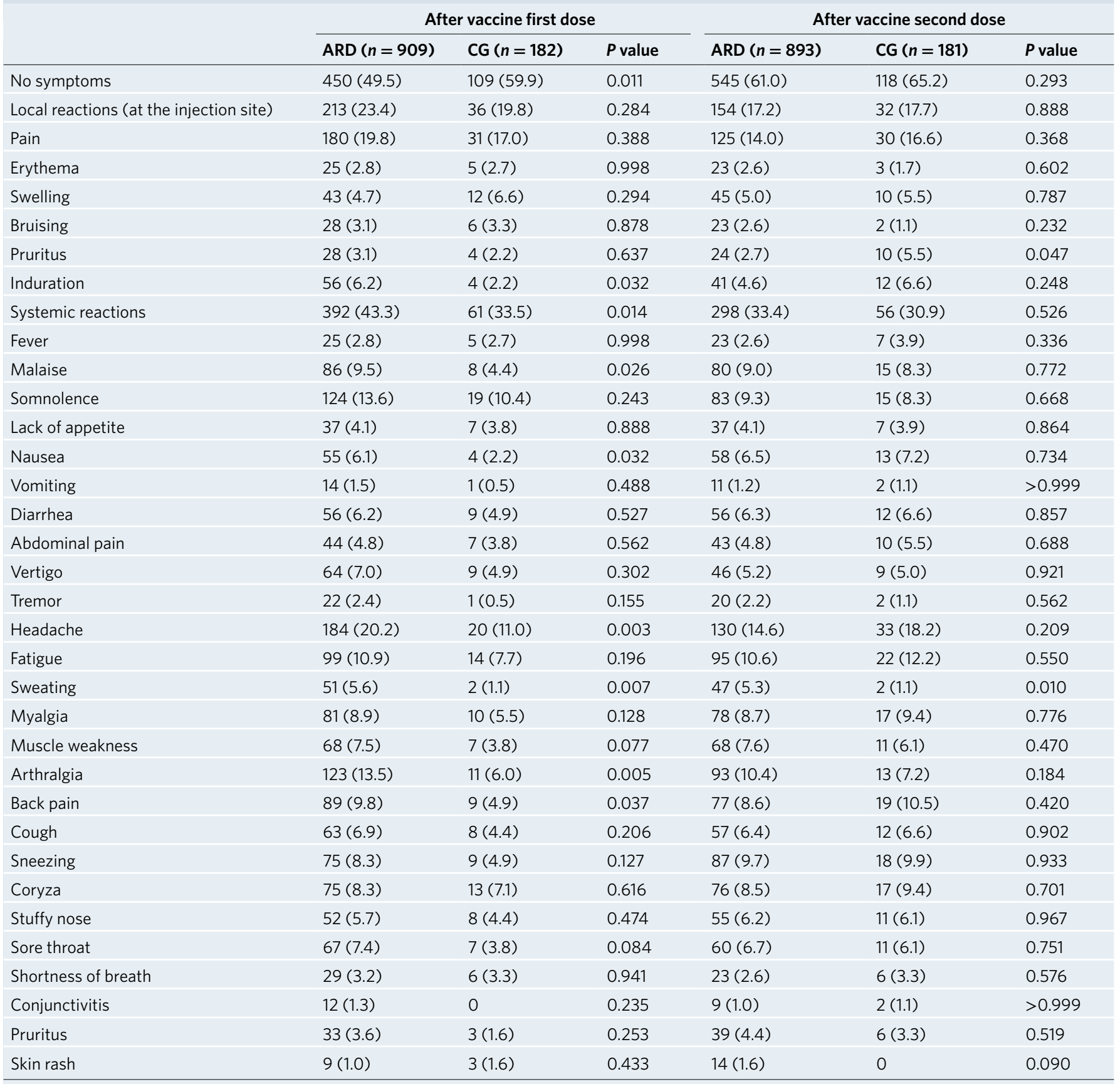

Results are presented as $n$ (\%) and compared with the chi-square or Fisher's exact test, as appropriate, always as two-sided analyses.

between D0 and D39 (until 10 days after the second dose) was higher compared to D40-D79 (33/1,092 (3.0\%) versus 6/1,057 (0.6\%), $P<0.0001)$. Four patients with ARD were hospitalized $(<10$ days after the second dose) and none died from COVID-19. There was no hospitalizations or deaths associated with COVID-19 in the CG. Eighteen symptomatic participants with RT-PCR-confirmed COVID-19 were genotyped in our service; $83.3 \%$ of infections were due to Gamma variants, $5.6 \%$ to Alpha and $11.1 \%$ to other variants. SARS-CoV-2 genotyping could not be performed in the remaining 21 symptomatic participants because they were unable to attend our center due to the long traveling distance involved, and therefore their samples were collected for RT-PCR at an independent laboratory near to their home.
Finally, we considered environmental factors that could influence SARS-CoV-2 infection risk in those participants who answered the targeted questions about their exposure. Patients with ARD reported higher adherence to social isolation 69.5 versus $21.7 \%$, $P<0.001)$ with lower household contact with infected people (4.6 versus $15.5 \%, P=0.0001)$ and lower use of public transportation (47.7 versus $81.7 \%, P<0.001$ ) compared to CG. The numbers of people living in the same home were comparable in both groups (median of two).

\section{Discussion}

Vaccination of immunosuppressed patients, who were excluded from phase 3 vaccine trials, is of the utmost importance since 
Table 5 | Baseline characteristics of patients with ARD with and without SC for anti-SARS-CoV-2 S1/S2 IgG antibodies and with and without NAb after two doses of CoronaVac vaccination

\begin{tabular}{|c|c|c|c|c|c|c|}
\hline & $\begin{array}{l}\text { ARD patients without } \\
\text { SC }(n=254)\end{array}$ & $\begin{array}{l}\text { ARD patients with } \\
\text { SC }(n=605)\end{array}$ & $P$ value & $\begin{array}{l}\text { ARD patients without } \\
\text { NAb }(n=375)\end{array}$ & $\begin{array}{l}\text { ARD patients with } \\
\text { NAb }(n=484)\end{array}$ & $P$ value \\
\hline \multicolumn{7}{|l|}{ Demographics } \\
\hline Current age (years) & $53(45-63)$ & $49(39-59)$ & $<0.001$ & $52(43-62)$ & $49(39-59)$ & $<0.001$ \\
\hline Age $\geq 60$ years & 89 (35) & $142(23.5)$ & $<0.001$ & $122(32.5)$ & 109 (22.5) & 0.001 \\
\hline Female sex & $208(81.9)$ & $452(74.7)$ & 0.023 & $293(78.1)$ & $367(75.8)$ & 0.427 \\
\hline Caucasian race & $144(56.7)$ & $312(51.6)$ & 0.170 & $213(56.8)$ & $243(50.2)$ & 0.055 \\
\hline $\mathrm{CIA}$ & $126(49.6)$ & $304(50.2)$ & 0.864 & $200(53.3)$ & $230(47.5)$ & 0.091 \\
\hline Other ARD & $128(50.4)$ & $301(49.8)$ & & $175(46.7)$ & $254(52.5)$ & \\
\hline \multicolumn{7}{|l|}{ Current therapy } \\
\hline Prednisone & $142(55.9)$ & $188(31.1)$ & $<0.001$ & $185(49.3)$ & $145(30.0)$ & $<0.001$ \\
\hline Prednisone dose (mg) & $5(5-10)$ & $5(5-10)$ & 0.926 & $5(5-10)$ & $5(5-10)$ & 0.731 \\
\hline Immunosuppressive drugs & $208(81.9)$ & $330(54.5)$ & $<0.001$ & $272(72.5)$ & $266(55)$ & $<0.001$ \\
\hline Methotrexate & $88(34.6)$ & $131(21.7)$ & $<0.001$ & $114(30.4)$ & $105(21.7)$ & 0.004 \\
\hline Leflunomide & $37(14.6)$ & $84(13.9)$ & 0.793 & $57(15.2)$ & $64(13.2)$ & 0.409 \\
\hline Mycophenolate mofetil & $62(24.4)$ & $48(7.9)$ & $<0.001$ & $67(17.9)$ & $43(8.9)$ & $<0.001$ \\
\hline Azathioprine & $31(12.2)$ & $69(11.4)$ & 0.739 & $40(10.7)$ & $60(12.4)$ & 0.433 \\
\hline Tofacitinib & $3(1.2)$ & $15(2.5)$ & 0.301 & $10(2.7)$ & $8(1.7)$ & 0.304 \\
\hline Cyclophosphamide & $2(0.8)$ & $7(1.2)$ & $>0.999$ & $3(0.8)$ & $6(1.2)$ & 0.739 \\
\hline Tacrolimus & $4(1.6)$ & $6(1.0)$ & 0.493 & $4(1.1)$ & $6(1.2)$ & 0.815 \\
\hline Cyclosporine & $4(1.6)$ & $4(0.7)$ & 0.245 & $6(1.6)$ & $2(0.4)$ & 0.085 \\
\hline Rituximab & $11(4.3)$ & $8(1.3)$ & 0.006 & $15(4.0)$ & $4(0.8)$ & 0.002 \\
\hline Ustekinumab & $1(0.4)$ & $4(0.7)$ & $>0.999$ & $2(0.5)$ & $3(0.6)$ & 0.869 \\
\hline
\end{tabular}

Results are expressed as median (IQR) and $n$ (\%). Continuous data were compared using the Mann-Whitney U-test, and categorical variables with the chi-square or Fisher's exact test, as appropriate, always as two-sided analyses. SC defined as positive serology (IgG titer $\geq 15 \mathrm{AU} \mathrm{ml}^{-1}$ ) for anti-SARS-CoV-2 S1/S2 IgG antibodies after vaccination (Indirect ELISA, LIAISON SARS-CoV-2 S1/S2 IgG). Positivity for NAb defined as neutralizing activity $\geq 30 \%$ (cPass sVNT Kit).

patients with ARD have an increased risk of hospitalization for severe COVID-19 (refs. ${ }^{21,24}$ ). In this large prospective study of an inactivated SARS-CoV-2 vaccine in patients with ARD, CoronaVac demonstrated a good safety profile with no serious/moderate AEs related to the vaccine. The vaccine was immunogenic in patients with ARD, but at lower levels when compared to the CG. Controlling the groups for age was essential, since SC may be lower in the older population ${ }^{10}$, and this differentiates the current trial from earlier studies ${ }^{15-18}$.

We prospectively included a large population of patients with $A R D$ representing eight systemic diseases fulfilling their respective classification criteria, and followed all participants with scheduled face-to-face appointments, telephone, smartphone instant messaging and email contacts, which allowed a more precise monitoring of vaccine-induced AEs in this population. Tolerance and safety are a relevant concern for patients with $\mathrm{ARD}$, since they have an intrin- sic risk for thrombosis ${ }^{28}$, a rare complication reported for some of the new COVID-19 vaccines $^{29}$, and autoimmune/autoinflammatory manifestations, a problem with adjuvanted vaccines in this already predisposed population ${ }^{30}$. Similar to previous results from CoronaVac trials in healthy populations ${ }^{31}$, most vaccine-related AEs were mild with pain at the injection site being the most frequently reported. Interestingly, vaccine-related AEs, particularly systemic symptoms, were much less frequent in both ARD and CG than those reported with mRNA vaccines ${ }^{32,33}$. These data confirm the previously reported safety profile of Corona $\mathrm{Vac}^{11}$, and extend this finding to a large group of immunocompromised patients. Data on disease activity were not available due to the study design, with approximately $93 \%$ of participants vaccinated in a single center over 2 days, and therefore the influence of this factor on CoronaVac immunogenicity remains to be determined. The lack of assessment of vaccine T cell responses was another limitation of the present study ${ }^{34,35}$. 
The exclusion of seropositive participants and those with COVID-19 during the study period allowed a more accurate evaluation of the immunogenicity of CoronaVac. In addition, there was no difference in blood sample collection timing between the two groups because most participants received vaccine in the same timeframe, precluding the possible confounding nonlinear relationship between the elapsed time and the vaccine. We observed lower CoronaVac immunogenicity in patients with ARD, although within the immunologic response standards (SC rates and GMT) established by the European Medicine Agency and the Food and Drugs Administration recommendations for Emergency Use Authorization of pandemic vaccines ${ }^{36,37}$. The $70 \%$ SC rate was comparable to that obtained against the pandemic influenza A / H1N1 inactivated vaccine (approximately 63\%) ) $^{27}$, but lower than those reported for the SARS-CoV-2 mRNA vaccine in a very small ARD population ${ }^{17}$ and in a study with patients predominantly using cytokine inhibitors and with limited representation of systemic diseases ${ }^{16}$. There was a substantial increase in immune response parameters, including anti-SARS-CoV-2 IgG titers and SC and NAb positivity rates, only after the second dose, reinforcing the importance of the full vaccination schedule for optimal vaccine immunogenicity, particularly in the ARD group. Similar to the anti-SARS-CoV-2 IgG antibody response, the frequency of mean inhibitory neutralizing activity against SARS-CoV-2 (56.4\%) was reduced compared to controls and that reported after SARS-CoV-2 mRNA vaccination ${ }^{15,16}$. Again, the second dose was essential to achieving the maximum response for both groups, with a lower neutralization activity in ARD than in CG after the two vaccine doses. A recent report including 53 patients with RA who had received mRNA vaccines also emphasized the importance of a second dose to improve immunogenicity ${ }^{38}$.

The profile of tertiary hospital patients evaluated in this trial, with a high frequency being treated with immunosuppressive/ glucocorticoid/biological therapies, probably contributed to the reduced humoral response observed in the ARD group. In fact, $63 \%$ were on immunosuppressive therapy and more than one-third on prednisone and biologics. Of note, these three groups of drugs were identified as independent variables that negatively impact both anti-SARS-CoV-2 IgG and neutralizing antibodies following vaccination. Among the immunosuppressive drugs, methotrexate and mycophenolate mofetil had the greatest negative impact on immunogenicity whereas abatacept and rituximab were the most negative among those treated with biologics. This finding is in line with other studies in patients with ARD and on other COVID vaccines $^{15,17,18,39}$ although these earlier reports did not control for age, which may limit the strength of the conclusions that can be drawn regarding the impact of these drugs ${ }^{18}$. Specifically for CoronaVac, these data added new information since another small trial found rituximab to be the only drug associated with low seropositivity after complete vaccination in immunocompromised patients ${ }^{19}$. We also found a detrimental effect of TNFi therapy solely on anti-S1/S2 IgG response, contrasting with a recent study of patients with $\mathrm{ARD}^{16}$. However, our findings require further investigation since most patients with CIA under TNFi were also being treated with methotrexate, which itself was associated with reduced humoral responses in the present trial.

Although not the main objective of this study, these data also provide preliminary evidence of the short-term efficacy of CoronaVac in prevention of symptomatic COVID-19 cases. An extension period of observation (up to 12 months) for incident cases is already in progress. Importantly, the majority of patients with $A R D$ and CG were all vaccinated at the same epidemiological week over a 2-day period, providing a unique setting of comparable influence of the ongoing local SARS-CoV-2 infection rates. Remarkably, the $45 \%$ increase in COVID-19 cases in Sao Paulo occurred from mid-March through to the end of April, coinciding with the study period between D40 and D79 ( $>10$ days after the second dose) ${ }^{40}$.
In this 40-day interval in which vaccine immunity is already expected, the frequency of COVID-19 cases was notably lower than in the previous 40 days after the first vaccination (D0-D39). The unanticipated overall similar frequency of SARS-CoV-2 infection in patients with ARD, a known vulnerable immunosuppressed population, compared to CG during the study period may be explained by the higher adherence to social isolation and lower household contact with infected people, as well as by reduced use of public transportation among patients. It may also be related to high exposure due to the professions of the majority of CG. The small number of new RT-PCR-confirmed COVID-19 cases during the observation period hampers, however, a definitive conclusion on the role of vaccine efficacy. The Gamma variant was the dominant strain amongst incident cases, in line with the virologic surveillance in the region, where Gamma represented $90 \%$ of all sequenced samples in the state in late April 2021 followed by Alpha and Beta as the other $\mathrm{VOC}^{41}$.

In conclusion, this study provides evidence of safety and reduced, but acceptable, short-term immunogenicity of an inactivated SARS-CoV-2 vaccine in the ARD population. The impact of this diminished humoral response on long-term vaccine effectiveness is already ongoing, and it will also shed light on the persistence of CoronaVac-elicited immune responses and the need for a vaccine booster

\section{Online content}

Any methods, additional references, Nature Research reporting summaries, source data, extended data, supplementary information, acknowledgements, peer review information; details of author contributions and competing interests; and statements of data and code availability are available at https://doi.org/10.1038/ s41591-021-01469-5.

Received: 22 May 2021; Accepted: 15 July 2021;

Published online: 30 July 2021

\section{References}

1. WHO Coronavirus (COVID-19) Dashboard with vaccination data. WHO https://covid19.who.int (2021).

2. de Souza, W. M. et al. Epidemiological and clinical characteristics of the COVID-19 epidemic in Brazil. Nat. Hum. Behav. 4, 856-865 (2020).

3. Faria, N. R. et al. Genomics and epidemiology of the P.1 SARS-CoV-2 lineage in Manaus, Brazil. Science 372, 815-821 (2021).

4. Coutinho, R. M. et al. Model-based estimation of transmissibility and reinfection of SARS-CoV-2 P. 1 variant. Preprint at https://www.medrxiv. org/content/10.1101/2021.03.03.21252706v (2021).

5. Funk, T. et al. Characteristics of SARS-CoV-2 variants of concern B.1.1.7, B.1.351 or P.1: data from seven EU/EEA countries, weeks 38/2020 to 10/2021. Eurosurveillance 26, 2100348 (2021)

6. Coronavírus Brasil. Painel coronavírus. Ministério da Saúde https://covid. saude.gov.br/ (2021).

7. Bueno, S. M. et al. Interim report: safety and immunogenicity of an inactivated vaccine against SARS-CoV-2 in healthy Chilean adults in a phase 3 clinical trial. Preprint at https://www.medrxiv.org/content/10.1101/ 2021.03.31.21254494v1 (2021).

8. Dizeres de texto de bula - Professional da Saude. Instituto Batantan https:// vacinacovid.butantan.gov.br/assets/arquivos/Bulas_Anvisa/2021.04.23 Bula profissional da saúde.pdf (2021).

9. Dejnirattisai, W. et al. Antibody evasion by the P.1 strain of SARS-CoV-2. Cell 184, 2939-2954 (2021).

10. Palacios, R. et al. Efficacy and safety of a COVID-19 inactivated vaccine in healthcare professionals in Brazil: the PROFISCOV Study. Preprint at https://papers.ssrn.com/sol3/papers.cfm?abstract_id=3822780 (2021).

11. Zhang, M.-X. et al. Safety of an inactivated SARS-CoV-2 vaccine among healthcare workers in China. Expert Rev. Vaccines https://www.tandfonline. com/doi/full/10.1080/14760584.2021 (2021).

12. Harpaz, R., Dahl, R. M. \& Dooling, K. L. Prevalence of immunosuppression among US adults, 2013. JAMA 316, 2547-2548 (2016).

13. Boyarsky, B. J. et al. Immunogenicity of a single dose of SARS-CoV-2 messenger RNA vaccine in solid organ transplant recipients. J. Am. Med. Assoc. 325, 1784-1786 (2021). 
14. Boyarsky, B. J. et al. Antibody response to 2-dose SARS-CoV-2 mRNA vaccine series in solid organ transplant recipients. J. Am. Med. Assoc. 325, 2204-2206 (2021).

15. Geisen, U. M. et al. Immunogenicity and safety of anti-SARS-CoV-2 mRNA vaccines in patients with chronic inflammatory conditions and immunosuppressive therapy in a monocentric cohort. Ann. Rheum. Dis. https://ard.bmj.com/content/early/2021/05/11/annrheumdis-2021$220272(2021)$

16. Simon, D. et al. SARS-CoV-2 vaccination responses in untreated, conventionally treated and anticytokine-treated patients with immunemediated inflammatory diseases. Ann. Rheum. Dis. https://ard.bmj.com/ content/early/2021/05/05/annrheumdis-2021-220461 (2021).

17. Boyarsky, B. J. et al. Antibody response to a single dose of SARS-CoV-2 mRNA vaccine in patients with rheumatic and musculoskeletal diseases. Ann. Rheum. Dis. https://ard.bmj.com/content/80/8/1098 (2021).

18. Furer, V. et al. Immunogenicity and safety of the BNT162b2 mRNA COVID-19 vaccine in adult patients with autoimmune inflammatory rheumatic diseases and in the general population: a multicentre study. Ann. Rheum. Dis. https://ard.bmj.com/content/early/2021/06/13/ annrheumdis-2021-220647 (2021).

19. Seyahi, E. et al. Antibody response to inactivated COVID-19 vaccine (CoronaVac) in immune-mediated diseases: a controlled study among hospital workers and elderly. Rheumatol. Int. 41, 1429-1440 (2021).

20. Grainger, R., Machado, P. M. \& Robinson, P. C. Novel coronavirus disease-2019 (COVID-19) in people with rheumatic disease: epidemiology and outcomes. Best Pract. Res. Clin. Rheumatol. 35, 101657 (2021).

21. Gianfrancesco, M. et al. Characteristics associated with hospitalisation for COVID-19 in people with rheumatic disease: data from the COVID-19 Global Rheumatology Alliance physician-reported registry. Ann. Rheum. Dis. 79, 859-866 (2020).

22. Pablos, J. et al. Clinical outcomes of patients with COVID-19 and chronic inflammatory and autoimmune rheumatic diseases: a multicentric matched-cohort study. Ann. Rheum. Dis. 79, 1544-1549 (2020).

23. Mikuls, T. R. et al. American College of Rheumatology Guidance for the Management of Rheumatic Disease in Adult Patients During the COVID-19 Pandemic: Version 3. Arthritis Rheumatol. 73, e1-e12 (2021).

24. Strangfeld, A. et al. Factors associated with COVID-19-related death in people with rheumatic diseases: results from the COVID-19 Global Rheumatology Alliance physician-reported registry. Ann. Rheum. Dis. 80, 930-942 (2021).

25. Avanzato, V. A. et al. Case study: prolonged infectious SARS-CoV-2 shedding from an asymptomatic immunocompromised individual with cancer. Cell 183, 1901-1912 (2020)

26. Choi, B. et al. Persistence and evolution of SARS-CoV-2 in an immunocompromised host. N. Engl. J. Med. 383, 2291-2293 (2020).

27. Saad, C. G. S. et al. Immunogenicity and safety of the 2009 non-adjuvanted influenza $\mathrm{A} / \mathrm{H} 1 \mathrm{~N} 1$ vaccine in a large cohort of autoimmune rheumatic diseases. Ann. Rheum. Dis. 70, 1068-1073 (2011).
28. Lee, J. J. \& Pope, J. E. A meta-analysis of the risk of venous thromboembolism in inflammatory rheumatic diseases. Arthritis Res. Ther. 16, 435 (2014).

29. Hunter, P. R. Thrombosis after covid-19 vaccination. Br. Med. J. 16, n958 (2021).

30. Shoenfeld, Y. \& Agmon-Levin, N. 'ASIA' - autoimmune/inflammatory syndrome induced by adjuvants. J. Autoimmun. 36, 4-8 (2011).

31. Zhang, Y. et al. Safety, tolerability, and immunogenicity of an inactivated SARS-CoV-2 vaccine in healthy adults aged 18-59 years: a randomised, double-blind, placebo-controlled, phase 1/2 clinical trial. Lancet Infect. Dis. 21, 181-192 (2021).

32. Mulligan, M. J. et al. Phase I/II study of COVID-19 RNA vaccine BNT162b1 in adults. Nature 586, 589-593 (2020)

33. Baden, L. R. et al. Efficacy and safety of the mRNA-1273 SARS-CoV-2 vaccine. N. Engl. J. Med. 384, 403-416 (2021).

34. Barrett, J. R. et al. Phase $1 / 2$ trial of SARS-CoV-2 vaccine ChAdOx $1 \mathrm{nCoV}-19$ with a booster dose induces multifunctional antibody responses. Nat. Med. 27, 279-288 (2021).

35. Folegatti, P. M. et al. Safety and immunogenicity of the ChAdOx1 nCoV-19 vaccine against SARS-CoV-2: a preliminary report of a phase $1 / 2$, single-blind, randomised controlled trial. Lancet 396, 467-478 (2020).

36. Committee for Medicinal Products for Human Use (CHMP). Guideline on Risk Management Systems for Medicinal Products for Human Use (European Medicines Agency, 2018); https://www.ema.europa.eu/en/documents/ scientific-guideline/draft-guideline-clinical-evaluation-vaccinesrevision-1 en.pdf

37. Guidance for Industry: Clinical Data Needed to Support the Licensure of Pandemic Influenza Vaccines (US Department of Health and Human Services Food and Drug Administration Center for Biologics Evaluation and Research, 2007); https://www.fda.gov/files/vaccines, \%20blood\%20\&\%20biologics/ published/Guidance-for-Industry-Clinical-Data-Needed-to-Supportthe-Licensure-of-Pandemic-Influenza-Vaccines.pdf

38. Rubbert-Roth, A. et al. Anti-SARS-CoV-2 mRNA vaccine in patients with rheumatoid arthritis. Lancet Rheumatol. 3, e470-e472 (2021).

39. Spiera R., Jinich S., Jannat-Khah D. Rituximab, but not other antirheumatic therapies, is associated with impaired serological response to SARS- CoV-2 vaccination in patients with rheumatic diseases. Ann. Rheum. Dis. https://ard.bmj.com/content/early/2021/05/10/annrheumdis-2021220604 (2021)

40. Coronavírus - Boletim Completo. Governo do Estado de São Paulo https:// www.seade.gov.br/coronavirus/\# (2020).

41. Notícias Coronavírus: Variante P1 já responde por $90 \%$ das amostras em SP. saopaulo.sp.gov.br https://www.saopaulo.sp.gov.br/noticias-coronavirus/ governo-de-sp-conclui-estudo-sobre-variantes-do-novo-coronavirus-noestado/ (2021)

Publisher's note Springer Nature remains neutral with regard to jurisdictional claims in published maps and institutional affiliations.

(c) The Author(s), under exclusive licence to Springer Nature America, Inc. 202 


\section{Methods}

Ethics statement. The protocol was conducted according to the Declaration of Helsinki and local regulations, and approved by the National and Institutional Ethical Committee of Hospital das Clinicas HCFMUSP, Faculdade de Medicina, Universidade de Sao Paulo, Sao Paulo, SP, Brazil (no. CAAE: 42566621.0.0000.0068). Written informed consent was obtained from all participants before enrollment, including an agreement for sharing of source data following publication of this manuscript, with indirect identifiers. There was no participant compensation.

Study design. This phase 4 prospective controlled clinical trial (CoronavRheum clinicaltrials.gov, no. NCT04754698) was conducted at a single tertiary center in Brazil.

Patients and controls. Patients with ARD and $\geq 18$ years of age from the Outpatient Rheumatology Clinics at our center were included, with the following diagnoses: $\mathrm{RA}^{42}, \mathrm{SLE}^{43}, \operatorname{axSpA}^{44}, \mathrm{PsA}^{45}$, primary vasculitis ${ }^{46,47}, \mathrm{pSSj}^{48}, \mathrm{SSc}^{49}, \mathrm{IIM}^{50}$ and PAPS ${ }^{51}$

After confirmation of participation by patients with ARD, CG were invited, with frequency matching by age (up to \pm 5 years difference) and sex, using an Excel program for random selection of participants (one control/five patients). None of these were previously vaccinated in the hospital's regular campaign. ARD diagnosis, use of immunosuppressive drugs and HIV infection were exclusion criteria for CG, whereas other well-controlled medical conditions were allowed in the CG group (Extended Data Fig. 1). None of the patients included in this analysis held medications to improve vaccine response.

Overall exclusion criteria were: history of anaphylactic response to vaccine components; acute febrile illness or symptoms compatible with COVID-19 at vaccination; Guillain-Barré syndrome; decompensated heart failure (class III or IV); demyelinating disease; previous vaccination with any SARS-CoV-2 vaccine; history of live virus vaccine up to 4 weeks previously; inactivated viral vaccine up to 2 weeks previously; history of having received blood products up to 6 months before the study; individuals who did not agree to participate in the study; hospitalized patients; and prevaccination positive COVID-19 serology and/or $\mathrm{NAb}$ (for immunogenicity analysis) (Extended Data Fig. 1).

After receiving the first vaccine dose, participants with RT-PCR-confirmed COVID-19 were excluded from the immunogenicity analysis but included in the evaluation of incident cases.

Vaccination protocol. The vaccination protocol for patients with ARD and GC consisted of a two-dose schedule of the COVID-19 vaccine. The first dose (with blood collection) was given for most participants on 9-10 February 2021 (D0), the second dose (with blood collection) on 9-10 March 2021 (D28) and a final blood collection on 19 April 2021 (D69) at the Hospital Convention Center. Incident COVID-19 cases were assessed for a further 10 days until D79. This protocol was delayed by 4 weeks for participants with incident COVID-19 during the study. Ready-to-use syringes loaded with CoronaVac (Sinovac Life Sciences, batch no. 20200412 ), consisting of $3 \mu \mathrm{g}$ in $0.5 \mathrm{ml}$ of $\beta$-propiolactone-inactivated SARS-CoV-2 (derived from the CN02 strain of SARS-CoV-2 grown in African green monkey kidney cells-Vero 25 cells) with aluminum hydroxide as an adjuvant, were administered intramuscularly in the deltoid area.

Primary and secondary outcomes. The primary outcome was humoral immunogenicity assessed by two coprimary endpoints: the presence of anti-S1/S2 SARS-CoV-2 IgG and the presence of NAb 6 weeks after the second vaccine dose (D69).

Secondary immunogenicity outcomes were: anti-S1/S2 IgG seroconversion and the presence of NAb at D28 (after vaccine first dose); geometric mean titers of anti-S1/S2 IgG and their factor increase in GMT (FI-GMT) at D28 and D69; and median (IQR) neutralizing activity of NAb at D28 and D69.

A further secondary outcome was safety related to the vaccine doses. Additionally, factors associated with anti-SARS-CoV-2 IgG SC and NAb positivity and incident COVID-19 case evaluation were exploratory outcomes.

Samples for immunogenicity evaluation. To assess these outcomes, blood samples $(20 \mathrm{ml})$ from all participants were obtained at D0 (baseline, immediately before first vaccine dose), D28 (immediately before the second dose) and D69 (6 weeks after the second dose). Sera were stored in a freezer at $-70^{\circ} \mathrm{C}$.

Anti-SARS-CoV-2 S1/S2 IgG antibodies. A chemiluminescent immunoassay was used to measure human IgG antibodies against proteins S1 and S2 in the receptor-binding domain (RBD) (Indirect ELISA, LIAISON SARS-CoV-2 S1/ S2 IgG, DiaSorin). SC rate was defined as positive serology $\left(\geq 15.0 \mathrm{UA} \mathrm{ml}^{-1}\right)$ after vaccination, taking into consideration that only patients with prevaccination negative serology were included. GMT and 95\% CIs of these antibodies were also calculated at all time points, attributing the value of $1.9 \mathrm{UA} \mathrm{ml}^{-1}$ (half of the lower limit of quantification, $\left.3.8 \mathrm{UA} \mathrm{ml}^{-1}\right)$ to undetectable levels $\left(<3.8 \mathrm{UA} \mathrm{ml}^{-1}\right)$. FI-GMT is the ratio of GMT after vaccination to that before, with growth measured in titers. These values are also presented and compared as geometric means and $95 \%$ CIs.
SARS-CoV-2 cPass virus NAb. The SARS-CoV-2 sVNT Kit (GenScript) was utilized according to the manufacturer's instructions. This analysis detects circulating NAb against SARS-CoV-2 that block the interaction between the RBD of the viral Spike glycoprotein with the angiotensin-converting enzyme 2 cell surface receptor. Tests were performed on ETI-MAX-3000 equipment (DiaSorin). Samples were classified as either "positive" (inhibition $\geq 30 \%$ ) or "negative" (inhibition $<30 \%$ ), as suggested by the manufacturer ${ }^{52}$. The frequency of positive samples was calculated at all time points. Medians (IQR) of the percentage of neutralizing activity, for positive samples only, were calculated at all time points.

Vaccine AEs and incident cases of COVID-19. Safety was rigorously followed by the National Research Ethics Council, and all serious AEs were classified as either vaccine related or not related. In addition an independent Data Safety Monitoring Board, comprising vaccine-prominent experts, periodically reviewed and evaluated the study protocol. Patients and control groups were advised to report any side effects of the vaccine; to this end, they received on D0 (first dose) and D28 (second dose) a standardized diary for recording of local and systemic manifestations. Local manifestations included local pain, erythema, swelling, bruising, pruritus and induration at the vaccine site. Systemic reactions included fever, malaise, somnolence, lack of appetite, nausea, vomiting, diarrhea, abdominal pain, vertigo, tremor, headache, fatigue, myalgia, muscle weakness, arthralgia, back pain, cough, sneezing, coryza, stuffy nose, sore throat, shortness of breath, conjunctivitis, pruritus and skin rash. Vaccine AE severity was defined according to the WHO definition ${ }^{53}$

Environmental factors associated with high risk of exposure to SARS-CoV-2 were recorded from all participants, including adherence to social isolation, number of people living in the same house, household contact with infected people and use of public transportation.

Additionally, to evaluate incident COVID-19 cases (exploratory outcome), all patients with ARD and controls were instructed to communicate any manifestation associated or not with COVID-19 by telephone, smartphone instant messaging or email. Our medical team was divided to provide a proper follow-up for the assigned group of patients/controls including the need for medical care, hospitalizations, severity of infections, sick days and treatment. Participants with suspicion of COVID-19 were instructed to seek medical care near their residence and, if recommended, to come to our tertiary hospital to undergo a RT-PCR test for SARS-CoV-2 or make an in-person visit. If tertiary care was required, the participant was transferred to a referenced hospital. The standardized diary of AEs was carefully reviewed with each participant on the day of the second dose (D28) and at the last visit (D69). COVID-19 incident cases were followed for 40 days (from D0 to 10 days after the second dose (D39)) and thereafter for the following 40 days (from D40 to D79).

Study data were collected and managed using REDCap electronic data capture tools (10.5.0, 2021 Vanderbilt University) hosted at our Institution ${ }^{54,55}$.

RT-PCR for SARS-CoV-2 and analysis of VOC. Clinical samples for SARS-CoV-2 RT-PCR consisted of naso- and oropharyngeal swabs, using a laboratory-developed test ${ }^{56}$. All participants with positive test results were invited to collect samples at our hospital, and these materials were further analyzed for VOC. RNA was extracted using the QIAamp Viral RNA Mini Kit (Qiagen) according to the manufacturer's instructions. For rapid access of VOC, we performed two real-time PCR protocols in parallel. Romano et al. ${ }^{57}$ used two sets of probes to detect NSP6 $\Delta 106-108$, which encodes a protein that participates in the viral replication process and allows the differentiation of ancestral variants from Alpha, Beta and Gamma VOC. The protocol of Vogels et al. uses a multiplex quantitative RT-PCR (RT-qPCR) assay that targets three regions (N1, ORF1a $\Delta 3675-3677$ and Spike $\Delta 69-70$ primer) and facilitates differentiation of Alpha VOC from Beta and Gama VOC, and from ancestral variants ${ }^{58}$. To confirm the results, we sequenced the virus using a combination of targeted multiplex PCR amplification and a portable nanopore sequencing MinION platform (Oxford Nanopore Technologies) ${ }^{3,58}$. In brief, complementary DNA was synthesized with random hexamers and the Protoscript II First Strand cDNA synthesis Kit (New England Biolabs). Whole-genome multiplex PCR amplification was then conducted using the ARTIC network SARS-CoV-2 V3 primer scheme. Multiplex PCR products were purified using AmpureXP beads (Beckman Coulter), and quantification was carried out using the Qubit dsDNA High Sensitivity assay on the Qubit 3.0 (Life Technologies). Samples were then normalized (10 ng per sample), DNA fragments were barcoded using the EXP-NBD104 (refs. ${ }^{59,60}$ ) and EXP-NBD114 (ref. ${ }^{61}$ ) Native Barcoding Kits (Oxford Nanopore Technologies) and pooled. Sequencing adapter ligation was performed using the SQK-LSK 109 Kit (Oxford Nanopore Technologies). Sequencing libraries were loaded onto an R9.4.1 flow-cell (Oxford NanoporeTechnologies) and sequenced using MinKNOW v.20.10.3 (Oxford Nanopore Technologies).

Symptomatic participants who were unable to come to our center to collect the RT-PCR kit were instructed to go to an independent laboratory near their home.

Statistical analysis. Sample size calculation was based on the previous $15 \%$ reduction in SC rate after first vaccination with the 2009 non-adjuvanted influenza $\mathrm{A} / \mathrm{H} 1 \mathrm{~N} 1$ vaccine in a large cohort of patients with $\mathrm{ARD}^{36}$. In expectation of 
SC rates of $63 \%$ in the ARD patient cohort and $78 \%$ in the control group, and considering an alpha error of $5 \%$ and power of $80 \%$ in a $5 / 1$ ratio to include more patients with ARD, the minimum sample required would be 445 patients with ARD and 89 healthy subjects, sex controlled and of similar age. In expectation of a higher SC rate of $98 \%$ for this vaccine ${ }^{28}$, such sample size had a power $>99 \%$ to detect a 15\% reduction in SC of patients with ARD. Due to the peak of the ongoing pandemic in Brazil during the vaccination period, we invited additional patients and controls, expecting a high incidence of previously infected people and a high rate of infection.

Categorical variables are presented as number (percentage) and compared using the chi-square or Fisher's exact test, as appropriate. Only for patients with ARD, multivariate logistic regression analyses were performed using as dependent variables SC or the presence of NAb at D69 (primary endpoints), and as independent variables those with $P<0.2$ in each univariate analysis.

Continuous general data are presented as medians (IQR) and compared using the Mann-Whitney $U$-test for intergroup comparison. Continuous data regarding anti-S1/S2 serology titers are presented as geometric means ( $95 \% \mathrm{CI})$; their comparisons were performed using repeated-measures analysis of variance (ANOVA) with two factors (two groups (ARD and CG) at three time points (D0, D28 and D69)), followed by Bonferroni's multiple comparisons in ln-transformed data.

Statistical significance was defined as $P<0.05$. All statistical analyses were performed using Statistical Package for the Social Sciences, v.20.0 (IBM-SPSS for Windows 20.0)

Reporting Summary. Further information on research design is available in the Nature Research Reporting Summary linked to this article.

\section{Data availability}

All background information on controls and clinical information for patients with ARD in this study are included in the Source data provided with this paper (https://figshare.com/s/0a8921e7422a4fb8436f). Requests for sera sharing will need approval from the Hospital das Clinicas da Universidade de Sao Paulo's review board and the National Research Ethics Council and a Material Transfer Agreement, which typically requires about 1 month. The SARS-CoV-2 sequences are available on GISAID (http://www.gisaid.org) (nos. EPI_ISL_28948692894885). An account (free registration) on GISAID is needed to obtain access to sequences. Additional correspondence and requests for materials should be addressed to the corresponding author (E.B.)

\section{References}

42. Aletaha, D. et al. 2010 Rheumatoid arthritis classification criteria: an American College of Rheumatology/European League against Rheumatism collaborative initiative. Arthritis Rheum. 62, 2569-2581 (2010).

43. Petri, M. et al. Derivation and validation of the Systemic Lupus International Collaborating Clinics classification criteria for systemic lupus erythematosus. Arthritis Rheum. 64, 2677-2686 (2012).

44. Rudwaleit, M. et al. The development of Assessment of SpondyloArthritis International Society classification criteria for axial spondyloarthritis (part II): validation and final selection. Ann. Rheum. Dis. 68, 777-783 (2009).

45. Tillett, W. et al. The ClASsification for Psoriatic ARthritis (CASPAR) Criteria - a retrospective feasibility, sensitivity, and specificity study. J. Rheumatol. 39, 154-156 (2012).

46. Arend, W. P. et al. The American College of Rheumatology 1990 criteria for the classification of takayasu arteritis. Arthritis Rheum. 33, 1129-1134 (2010).

47. Leavitt, R. Y. et al. The American College of Rheumatology 1990 criteria for the classification of Wegener's granulomatosis. Arthritis Rheum. 33 1101-1107 (2010).

48. Vitali, C. Classification criteria for Sjogren's syndrome: a revised version of the European criteria proposed by the American-European Consensus Group. Ann. Rheum. Dis. 61, 554-558 (2002).

49. van den Hoogen, F. et al. 2013 classification criteria for systemic sclerosis: an American College of Rheumatology/European League against Rheumatism collaborative initiative. Ann. Rheum. Dis. 72, 1747-1755 (2013).

50. Lundberg, I. E. et al. EULAR/ACR classification criteria for adult and juvenile idiopathic inflammatory myopathies and their major subgroups. Ann. Rheum. Dis. 76, 1955-1964 (2017).

51. Miyakis, S. et al. International consensus statement on an update of the classification criteria for definite antiphospholipid syndrome (APS). J. Thromb. Haemost. 4, 295-306 (2006).

52. Taylor, S. C. et al. A new SARS-CoV-2 dual-purpose serology test: highly accurate infection tracing and neutralizing antibody response detection. J. Clin. Microbiol. 59, 4 (2021).
53. WHO Draft Guidelines for Adverse Event Reporting and Learning Systems (WHO, 2021); https://apps.who.int/iris/bitstream/handle/10665/69797/ WHO-EIP-SPO-QPS-05.3-eng.pdf? sequence $=1$ \&isAllowed $=y$

54. Harris, P. A. et al. The REDCap consortium: building an international community of software platform partners. J. Biomed. Inform. 95, 103208 (2019)

55. Harris, P. A. et al. Research electronic data capture (REDCap) - a metadata-driven methodology and workflow process for providing translational research informatics support. J. Biomed. Inform. 42, 377-381 (2009)

56. Corman, V. M. et al. Detection of 2019 novel coronavirus (2019-nCoV) by real-time RT-PCR. Eurosurveillance 25, 2000045 (2020).

57. Romano, C. M. et al. Real-time PCR protocol to screen for SARS-COV-2 variants of concern (B. 1.1. 7, P. 1 and B. 1.1. 35). protocols.io https://www. protocols.io/view/real-time-pcr-protocol-to-screen-for-sars-cov-2-vabszbnf2n (2021)

58. Vogels, C. B. F. et al. Multiplex qPCR discriminates variants of concern to enhance global surveillance of SARS-CoV-2. PLoS Biol. 19, e3001236 (2021).

59. Hallal, P. C. et al. SARS-CoV-2 antibody prevalence in Brazil: Results from two successive nationwide serological household surveys. Lancet Glob. Health 8, e1390-e1398 (2020)

60. Buss, L. F. et al. Three-quarters attack rate of SARS-CoV-2 in the Brazilian Amazon during a largely unmitigated epidemic. Science 371, 288-292 (2021)

61. Rambaut, A. et al. Preliminary genomic characterisation of an emergent SARS-CoV-2 lineage in the UK defined by a novel set of spike mutations. Virological.org https://virological.org/t/preliminary-genomic characterisation-of-an-emergent-sars-cov-2-lineage-in-the-uk-definedby-a-novel-set-of-spike-mutations/563 (2020).

\section{Acknowledgements}

We thank the contribution of the Central Laboratory Division, Registry Division, Security Division, IT Division, Superintendence, Pharmacy Division and Vaccination Center for their technical support. We also thank the volunteers for participating in the three in-person visits of the protocol and for handling the biological material, and those responsible for the follow-up of all participants. This study was sponsored by grants from Fundação de Amparo à Pesquisa do Estado de São Paulo (no. 2015/03756-4 to N.E.A., S.G.P., C.A.S. and E.B.; no. 2017/14352-7 to T.P., no. 2018/09937-9 to V.A.O.M., no. 2020/11677-5 to G.B.H.D.; and no. 2019/21173-7 to C.T.R.) and from Conselho Nacional de Desenvolvimento Científico e Tecnológico (no. 305242/2019-9 to E.B.; no. 304984/2020-5 to C.A.S.; no. 305556/2017-7 to R.M.R.P.; and no. 303379/2018-9 to S.K.S.). Instituto Butantan supplied the study product and had no other role in the trial.

\section{Author contributions}

A.C.M.-R., N.E.A., C.G.S., E.F.N.Y., T.P., S.G.P., E.G.K. and E.B. conceived and designed the study, participated in data collection and analysis and supervised clinical data management, writing of the manuscript and revision of the manuscript. S.G.R.F. and P.T.R. organized and supervised blood collection and vaccination. A.J.S.D. and L.A. supervised serum processing, SARS-CoV-2-specific antibody ELISA/neutralization assays and SARS-CoV-2 RT-PCR. A.C.M.-R., N.E.A., C.G.S., E.F.N.Y., T.P., S.G.P., E.B, S.R.G.F., P.T.R., R.M.R.P., S.K.S., D.C.O.A., P.D.S.-B., C.T.R., G.B.H.D., V.A.O.M. and C.A.S. collected epidemiological and clinical data and assisted with the identification of SARS-CoV-2 infection and follow-up of patients. M.H.L. organized and supervised the vaccination protocol. E.C.S. performed SARS-CoV-2 genotyping of positive RT-qPCR samples and screening of variants of concern. All authors helped to edit the manuscript.

\section{Competing interests}

The authors declare no competing interests.

\section{Additional information}

Extended data is available for this paper at https://doi.org/10.1038/s41591-021-01469-5.

Supplementary information The online version contains supplementary material available at https://doi.org/10.1038/s41591-021-01469-5.

\section{Correspondence and requests for materials should be addressed to E. B.}

Peer review information: Nature Medicine thanks Dawn Teare, Philip Robinson and the other, anonymous, reviewer(s) for their contribution to the peer review of this work. Saheli Sadanand was the primary editor on this article and managed its editorial process and peer review in collaboration with the rest of the editorial team.

Reprints and permissions information is available at www.nature.com/reprints. 
Autoimmune rheumatic diseases (ARD) patients

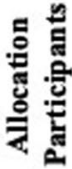

Excluded ( $n-225)$

-COVID-19 suspicion at D0 or RT-

PCR confirmed COVID-19 $<4$

weeks before $D 0(n=24)$

-Demyelinating disease $(n=1)$

-Previous SARS-Cov-2

vaccination $(n=25)$

-Inactivated virus vaccine

$<2$ weeks before $D 0(n=1)$

- Refused to participate $(n=161)$

- Hospitalized patients $(n=13)$

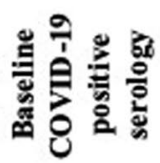

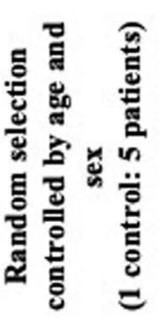

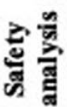

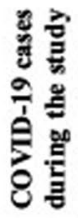

롤
Assessed for eligibility $(\mathrm{n}=1418)$ anti-S1/S2 IgG serology and/or neutralizing antibodies (NAb) $(\mathrm{n}=232)$ $(n=51)$
Control group (CG)

Assessed for eligibility $(\mathrm{n}=542)$ - Refused to participate ( $n=50)$

Excluded due to positive baseline antiS1/S2 IgG serology and/or neutralizing antibodies (NAb) $(\mathrm{n}=191)$

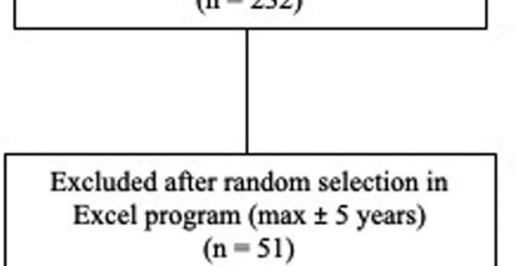

Excluded after random selection in Excel program ( $\max \pm 5$ years) $(\mathrm{n}=119)$

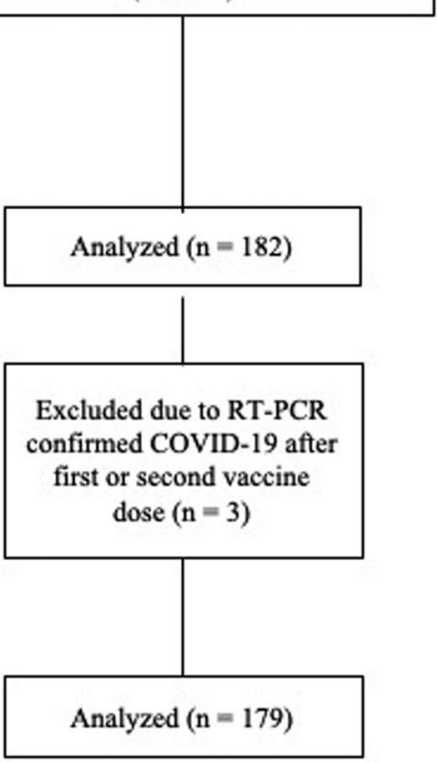

Extended Data Fig. 1 | Trial Design. The diagram depicts the enrollment and analysis of participants in the ARD and CG groups. Reasons for exclusions are provided. 


\section{Reporting Summary}

Nature Research wishes to improve the reproducibility of the work that we publish. This form provides structure for consistency and transparency in reporting. For further information on Nature Research policies, see our Editorial Policies and the Editorial Policy Checklist.

\section{Statistics}

For all statistical analyses, confirm that the following items are present in the figure legend, table legend, main text, or Methods section.

$\mathrm{n} / \mathrm{a}$ Confirmed

$\bigotimes$ The exact sample size $(n)$ for each experimental group/condition, given as a discrete number and unit of measurement

$\bigotimes$ A statement on whether measurements were taken from distinct samples or whether the same sample was measured repeatedly

$\triangle$ The statistical test(s) used AND whether they are one- or two-sided

Only common tests should be described solely by name; describe more complex techniques in the Methods section.

$\bigotimes$ A description of all covariates tested

Х $\square$ A description of any assumptions or corrections, such as tests of normality and adjustment for multiple comparisons

$\square$ A full description of the statistical parameters including central tendency (e.g. means) or other basic estimates (e.g. regression coefficient) AND variation (e.g. standard deviation) or associated estimates of uncertainty (e.g. confidence intervals)

For null hypothesis testing, the test statistic (e.g. $F, t, r$ ) with confidence intervals, effect sizes, degrees of freedom and $P$ value noted Give $P$ values as exact values whenever suitable.

Х $\square$ For Bayesian analysis, information on the choice of priors and Markov chain Monte Carlo settings

Х $\square$ For hierarchical and complex designs, identification of the appropriate level for tests and full reporting of outcomes

$\triangle \square$ Estimates of effect sizes (e.g. Cohen's $d$, Pearson's $r$ ), indicating how they were calculated

\section{Our web collection on statistics for biologists contains articles on many of the points above.}

\section{Software and code}

Policy information about availability of computer code

Data collection The analyzed data were extracted

from the patients' electronic medical records (PRONTMED) and Study data were collected and managed using REDCap electronic data capture tools (10.5.0 - (C) 2021 Vanderbilt University) hosted at our Institution.

Data collection for ELISA was performed using Indirect ELISA, LIAISON ${ }^{\circledR}$ SARS-CoV-2 S1/S2 IgG, DiaSorin, Italy. Neutralizing antibodies were performed on the ETI-MAX-3000 equipment (DiaSorin, Italy).

No custom software codes have been developed.

Data analysis All statistical analyses were performed using Statistical Package for the Social Sciences version 20.0 (IBM-SPSS for Windows. 20.0. Chicago, IL, USA)

For manuscripts utilizing custom algorithms or software that are central to the research but not yet described in published literature, software must be made available to editors and

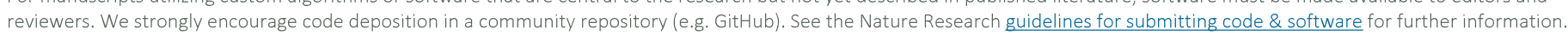

\section{Data}

Policy information about availability of data

All manuscripts must include a data availability statement. This statement should provide the following information, where applicable:

- Accession codes, unique identifiers, or web links for publicly available datasets

- A list of figures that have associated raw data

- A description of any restrictions on data availability

All the background information on controls and clinical information for the patients with ARD in this study are included in the Source Data provided with this paper (https://figshare.com/s/0a8921e7422a4fb8436f). Requests for sera sharing will need approval from the Hospital das Clinicas da Universidade de Sao Paulo's review 


\section{Field-specific reporting}

Please select the one below that is the best fit for your research. If you are not sure, read the appropriate sections before making your selection.

\ Life sciences

Behavioural \& social sciences

Ecological, evolutionary \& environmental sciences

For a reference copy of the document with all sections, see nature.com/documents/nr-reporting-summary-flat.pdf

\section{Life sciences study design}

All studies must disclose on these points even when the disclosure is negative.

Sample size The sample size calculation was based on the previous 15\% reduction of seroconversion rate after primo vaccination with the 2009 nonadjuvanted influenza A/H1N1 vaccine in a large cohort of ARD patients 35 . Expecting seroconversion rates of $63 \%$ in the ARD patient's cohort and $78 \%$ in the control group, considering an alpha error of $5 \%$ and power of $80 \%$, in $5: 1$ ratio in order to include more ARD patients, the minimum sample required would be 445 ARD patients and 89 healthy subjects, sex-matched and with similar ages.

Data exclusions All safety and immunogenicity data were included in the study. No data were excluded from the analyses.

Replication This is an ongoing human trial and therefore there was still no attempt of replication.

Randomization This was an observational study with no randomized intervention. All participants (patients and controls) received the same vaccine, without experimental groups.

Blinding This phase 4 prospective controlled observational study with no randomized intervention. All participants (patients and controls) received the same vaccine, without placebo group. Therefore, blinding was not performed.

\section{Reporting for specific materials, systems and methods}

We require information from authors about some types of materials, experimental systems and methods used in many studies. Here, indicate whether each material, system or method listed is relevant to your study. If you are not sure if a list item applies to your research, read the appropriate section before selecting a response.

\begin{tabular}{|c|c|}
\hline$n / a$ & Involved in the study \\
\hline Х & $\square$ Antibodies \\
\hline$\bigotimes$ & $\square$ Eukaryotic cell lines \\
\hline$\bigotimes$ & $\square$ Palaeontology and archaeology \\
\hline Х & $\square$ Animals and other organisms \\
\hline Г & \ Human research participants \\
\hline $\mathbf{L}^{-1}$ & $\bigotimes$ Clinical data \\
\hline$\bigotimes$ & $\square$ Dual use research of concern \\
\hline
\end{tabular}

\begin{tabular}{l|l} 
Methods \\
\hline n/a & Involved in the study \\
$\searrow$ & $\square$ ChIP-seq \\
$\square$ & $\square$ Flow cytometry \\
$\square$ & $\square$ MRI-based neuroimaging
\end{tabular}

\section{Human research participants}

Policy information about studies involving human research participants

Population characteristics

Recruitment
Male and female individuals with autoimmune rheumatic disease and volunteers (control group) $\geq 18$ anos. The 910 patients with ARD and 182 controls included in immunogenicioty analysis had comparable median ages [51 (40-60) vs. 50 (41-60) years, $p=0.985$ ] and female sex (76.9\% vs. $76.9 \%, p>0.999)$. Three hundred and forty-eight (38.2\%) patients were receiving ongoing treatment with prednisone, median dose 5 (5-10) $\mathrm{mg} /$ day, $573(63.0 \%)$ were using immunosuppressive drugs [methotrexate (25.2\%), leflunomide (14.3\%), mycophenolate mofetil (13.1\%), azathioprine (12\%) and others less than $3 \%$ each] and 321 (35.3\%) were under biologic therapy.

Autoimmune rheumatic disease (ARD) patients from the Outpatient Rheumatology Clinics at Hospital das Clinicas HCFMUSP, Faculdade de Medicina, Universidade de Sao Paulo, Sao Paulo, SP, Brazil. Control Group (CG) were invited, matching by gender and sex (up to \pm 5 years differences). None of them were previously vaccinated in the hospital's regular campaign. Well-controlled medical conditions were allowed in the CG, except ARD, use of immunosuppressive drugs or HIV infection. Overall exclusion criteria were: history of anaphylactic response to vaccine components, acute febrile illness or symptoms compatible to COVID-19 at vaccination, Guillain-Barré syndrome, decompensated heart failure (class III or IV), demyelinating disease, previous vaccination with any SARS-Cov-2 vaccine, history of live virus vaccine up to four weeks before, virus vaccine inactivated up to two weeks before, history of having received blood products up to six months before the study, individuals 
who did not accept to participate in the study, hospitalized patients, and pre-vaccination positive COVID-19 serology and/or neutralization antibodies.

All statistical analyses took into account the frequency matching, with exclusion of non-matched subjects. Immunogeniticy analysis also excluded incident COVID-19 cases and patients who did not attend the final visit, composing the final sample of 859 patients with ARD and 179 CG. In the logistic regression model (Supplementary Table 2, only with patients with ARD), the age was included in the model using the cut-off $>60$ years. This model intended to highlight the known importance of older age in vaccine response.

Ethics oversight

The protocol was conducted according to the Declaration of Helsinki and local regulations and approved by the National and Institutional Ethical Committee of Hospital das Clinicas HCFMUSP, Faculdade de Medicina, Universidade de Sao Paulo, Sao Paulo, SP, Brazil (CAAE: 42566621.0.0000.0068). Written informed consent was obtained from each participant before enrollment.

Note that full information on the approval of the study protocol must also be provided in the manuscript.

\section{Clinical data}

Policy information about clinical studies

All manuscripts should comply with the ICMJE guidelines for publication of clinical research and a completed CONSORT checklist must be included with all submissions.

Clinical trial registration

Study protocol

Data collection

Outcomes
ClinicalTrials.gov Identifier: NCT04754698

The protocolo has been submitted.The full trial can be assessed at ClinicalTrials.gov

The study was conducted at a single tertiary center in Brazil. Enrollment and vaccination occurred in the same day for each participant. The first subject was enrolled and vaccinated on Feb 9th, 2021 and the last participant was enrolled and vaccinated on February 24th, 2021. The vaccination protocol for patients with ARD and controls consisted of a two-dose schedule of the COVID-19 vaccine. The first dose with blood collection was given for most of participants on February 9-10th 2021 (D0), the second dose with blood collection on March 9-10th 2021 (D28) and the last blood collection on April 19th 2021 (D69) at the Hospital Convention Center. Incident COVID-19 cases were assessed for another 10 days to D79.

During 2 consecutive days of the 2021 epidemiological week 6th, all ARD patients and CG received the 1st CoronaVac dose, repeated at a 2-dose schedule after 28 days. Blood samples were collected from all participants for quantitative serological testing for SARS$\mathrm{CoV}$-2. The primary outcome was seroconversion rate (SC) at 6 weeks after the 2 nd dose. Geometric meantitles (GMT) and factor increase in GMT (FI-GMT) were also calculated. ARD patients and CG were evaluated using standardized vaccination and COVID-19 symptom diaries, 3 face-to-face visits, and 24-hs available phone, whatsapp and e-mail contact. Symptomatic cases were tested by RT-PCR for SARS-CoV-2 and a subgroup of positive samples were evaluated for the presence of variants of concerns (Gamma, Alpha and Beta lineages).

Immunogenicity and safety of the CoronaVac vaccine in ARDs patients.

Primary Outcome Measure: presence of $\geq 30 \%$ of neutralizing activity of SARS-CoV- 2 antibodies and seroconversion rate of anti-SARSCov-2 IgG antibodies.

Secondary outcome: safety of CoronaVac in a large cohort of ARD patients compared with age- and sex-matched controls without these conditions. Incident symptomatic cases confirmed by real-time reverse transcriptase-polymerase chain reaction (RT-PCR) for SARS-CoV-2 and the presence of variants of concerns (Gamma, Alpha and Beta lineages). 